\title{
Centralisation, décentralisation et accès aux services urbains : le cas de l'enlèvement des ordures ménagères à Abidjan
}

Centralization, decentralization, access to urban public services : waste collection in Abidjan

\section{Amandine Henry}

\section{(2) OpenEdition \\ Journals}

Édition électronique

URL : http://journals.openedition.org/belgeo/7333

DOI : $10.4000 /$ belgeo.7333

ISSN : 2294-9135

\section{Éditeur :}

National Committee of Geography of Belgium, Société Royale Belge de Géographie

Édition imprimée

Date de publication : 31 décembre 2009

Pagination : 425-438

ISSN : 1377-2368

\section{Référence électronique}

Amandine Henry, "Centralisation, décentralisation et accès aux services urbains : le cas de l'enlèvement des ordures ménagères à Abidjan », Belgeo [En ligne], 3-4 | 2009, mis en ligne le 17 mars 2013, consulté le 01 mai 2019. URL : http://journals.openedition.org/belgeo/7333 ; DOI : 10.4000/ belgeo.7333

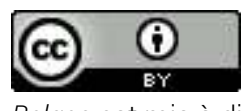

Belgeo est mis à disposition selon les termes de la licence Creative Commons Attribution 4.0 International. 


\title{
Centralisation, décentralisation et accès aux services urbains: le cas de l'enlèvement des ordures ménagères à Abidjan
}

\author{
Amandine Henry \\ Université Libre de Bruxelles
}

\begin{abstract}
RÉSUMÉ
Cet article traite de l'inégalité d'accès aux services publics urbains dans les pays en développement et, plus particulièrement, de la question de l'accès au service d'enlèvement des ordures ménagères à Abidjan. Cette question est étroitement liée au mouvement de centralisation, décentralisation et recentralisation qui a affecté la gestion de ce service au cours des dernières décennies. Chaque étape de l'avancée de la Côte d'lvoire dans le processus de décentralisation a provoqué des changements institutionnels dans la gestion des ordures ménagères à Abidjan; tous ont eu des effets spécifiques sur l'accès de la population à ce service. Ces effets sont étudiés à l'échelle des communes et des quartiers à travers une enquête de terrain, et sont liés aux caractéristiques socio-économiques des quartiers. Une analyse comparative entre deux communes de standing opposé, ainsi que l'examen des aspects historiques de la problématique, s'attachent à identifier les facteurs-clés d'une telle inégalité d'accès au service d'enlèvement des ordures ménagères à Abidjan.
\end{abstract}

MOTS-CLÉS: décentralisation, services publics, services urbains, enlèvement des ordures, gestion des ordures, accès aux services, fragmentation urbaine, Côte d'Ivoire

\begin{abstract}
CENTRALIZATION, DECENTRALIZATION, ACCESS TO URBAN PUBLIC SERVICES: WASTE COLLECTION IN ABIDJAN

This paper deals with the inequality of access to the urban public utilities in developing countries, and more accurately with the issue of the access to the waste collection in Abidjan. This question is closely linked with the centralization, decentralization and recentralization movements that affected this service management for the last decades. Each stage of the decentralization process in Ivory Coast brought institutional changes in waste collection management in Abidjan. Each of them had specific consequences on the access of people to this service. These consequences are investigated on a local scale and are linked to the socio-economic characteristics of the studied areas. Together with the focus on the historical aspects of the problematic, a comparative analysis between two districts of opposite standing attempts to identify the key factors of such an inequity of access to the waste collection service in Abidjan.
\end{abstract}

KEY WORDS: decentralization, public services, urban services, waste collection, waste management, access to services, splintering urbanism, Ivory Coast 


\section{INTRODUCTION}

a question de l'accès aux services urbains dans les pays en développement doit être envisagée dans un contexte d'espace urbain étalé et ségrégé depuis ses origines, en croissance rapide et globalement pauvre. Les réformes libérales accompagnant les programmes d'ajustements structurels (PAS) introduits sous la pression des bailleurs de fonds dans les années 80 ont eu des conséquences profondes sur l'accès aux services urbains. D'abord, le mode de financement de réseaux coûteux et centralisés (eau, assainissement, électricité, enlèvement des ordures...) a mené fréquemment au phénomène paradoxal selon lequel la population aisée du centre bénéficie de services subventionnés alors que les populations périphériques paupérisées en paient l'accès au prix fort (Couret et Metzger, 2002). Mais surtout, ces réformes ont orienté les modalités de gestion et de développement des services urbains: remise en cause de l'idéal du «service universel» au profit du «service pour le plus grand nombre», réduction des dépenses publiques par un désengagement de l'Etat, introduction de logiques marchandes (recouvrement des coûts, privatisations...) endogénéisées ensuite par les autorités locales (Dorier-Apprill et Jaglin, 2002).

Marvin et Graham (2001) vont plus loin dans leur thèse de la fragmentation urbaine (splintering urbanism). Selon eux, alors que les services urbains en réseau ont contribué à une intégration intra-urbaine croissante au cours du XXème siècle, les réformes libérales amenées par les PAS ont entraîné la rupture du contrat social qui assurait la cohésion des sociétés urbaines, en mettant en question l'idéal moderne des services universels. Cela a engendré le développement de pratiques de contournement sélectif des zones les moins solvables et de desserte de qualité pour les élites dans des espaces de première classe déconnectés du tissu urbain d'ensemble (type gated communities par exemple). C'est en ce sens que, d'après ces auteurs, les réformes dans les services ont produit un espace urbain fragmenté. La notion de fragmentation dépasse ici celle d'inégalités socio-spatiales, qui désigne l'inscription dans l'espace des inégalités socio-économiques, car celle-là est une notion multidimensionnelle qui indique la faiblesse des liens politiques, sociaux (ségrégation résidentielle, replis communautaristes), économiques, spatiaux (déconnexions, discontinuités physiques), fiscaux, etc. entre différentes portions de l'espace urbain.

L'accent est ensuite mis, dans les politiques de coopération internationale, sur l'ajustement politique, sans lequel toute forme d'aide semble inefficace. Apparaît alors le concept de «bonne gouvernance», rapidement entendu au sens de démocratisation. La lenteur des changements dans ce sens conduit les instances internationales à contourner les Etats et trouver d'autres partenaires. C'est le début de l'engagement de nombreux pays en développement dans des processus de décentralisation, visant à transférer progressivement les responsabilités de l'Etat central à un niveau plus local. Cette optique trouve un écho important en Côte d'Ivoire. A la fin des années '60, les disparités régionales sont très importantes et le pays est fortement polarisé par Abidjan, qui concentre la majorité des équipements financés sur fonds publics, afin d'encourager les investissements étrangers. Ce déséquilibre régional constitue une menace particulière au vu des questions ethniques qui s'y surimposent. Dès lors, l'enjeu d'une décentralisation ivoirienne, outre celui de capter les aides des bailleurs internationaux, qui cherchent d'autres interlocuteurs que l'Etat pour les politiques 
de développement, est donc de préserver la stabilité politique et économique du pays. A la fin des années '70, avec l'aide des crédits internationaux, et pour combler les disparités de la période précédente, les autorités ivoiriennes mettent en place une politique de décentralisation, avec création de communes et de régions.

La ville d'Abidjan comporte une trame ségrégée et fonctionnalisée héritée de l'ère coloniale française. Celle-ci a ensuite été relayée par l'action structurante des sociétés immobilières publiques après l'Indépendance, dans le cadre du rêve du logement pour tous et de l'Etat comme promoteur et organisateur exclusif de l'espace urbain. L'espace résultant est profondément différencié. La ville, dont la population a été gonflée par la crise politique qui secoue le pays depuis 2002, connaît à présent des insuffisances profondes au niveau des services et des équipements en périphérie. Le service d'enlèvement des ordures ménagères est défaillant, et a été géré à différents niveaux de pouvoir au rythme de la décentralisation. Ainsi, chaque phase de gestion a provoqué des effets spécifiques sur la qualité de services et son homogénéité au sein de la ville. L'insalubrité d'Abidjan est aujourd'hui un problème crucial, tant pour les autorités que pour la population. Cela n'en fait pas une exception: la situation en matière de salubrité en Afrique sub-saharienne présente des constats alarmants. Si, en milieu rural, la question des déchets peut être résolue sans trop de nuisances par enfouissement ou combustion par les particuliers, le problème se pose avec autrement plus d'acuité dans les grandes villes, dans lesquelles croissance démographique et évolution des modes de consommation s'allient pour accroître toujours plus la quantité de déchets générés. Cela permet de comprendre l'ampleur du défi que représente la gestion des ordures pour les gouvernements urbains. Malgré les moyens financiers considérables déployés par les autorités pour la collecte des ordures - entre 20 et $50 \%$ des budgets municipaux en moyenne (Achankeng, 2003) - le taux de collecte dépasse rarement la moitié, voire $30 \%$ des déchets produits. Si bien que les amoncellements de déchets font à présent partie intégrante du paysage urbain africain et causent de graves dommages en matière de pollution de l'air, du sol, des eaux de surface et souterraines.

Les dysfonctionnements dans ce service sont différenciés spatialement: la collecte des ordures est bien souvent limitée aux centres urbains, vitrines du territoire national, alors que dans la périphérie, la collecte demeure absente ou limitée aux seules voies principales. Ainsi, à Libreville, le service d'enlèvement des déchets touche plus de la moitié des ménages dans les quartiers centraux, contre moins de $20 \%$ dans les quartiers périphériques (Mboumba, 2007). A Ouagadougou, alors que des entreprises privées assurent efficacement un service coûteux dans les quartiers résidentiels centraux, les habitants de la périphérie n'ont souvent d'autre alternative que le dépôt sauvage de leurs ordures où ils le peuvent, pratique qui concerne entre 65 et $90 \%$ des ménages de la périphérie (MeunierNikiema, 2007).

L'objectif de l'étude est d'analyser les inégalités socio-spatiales de la ville d'Abidjan en matière d'accès aux services publics, à travers le service d'enlèvements des ordures ménagères. A cet effet, nous tenterons de répondre aux questions suivantes: d'abord, l'accès au service d'enlèvement des ordures à Abidjan est-il réellement empreint d'inégalités socio-spatiales, et dans quelle mesure? Les quartiers périphériques défavorisés offrent-ils des services de qualité égale à ceux fournis dans le centre de la capitale? Si la différenciation spatiale de l'accès au service est établie, nous tenterons d'analyser la manière dont ces inégalités se sont mises en place sur les temps longs, dans le service d'enlèvement des ordures ménagères en particulier. II s'agit de voir si cette ségrégation spatiale a toujours existé, comment elle est apparue et a évolué à travers la succession des différentes périodes de gestion du service en parallèle avec le processus de décentralisation. Enfin, nous tenterons d'identifier les différents types de facteurs qui concourent à produire une telle diversification d'accès au service. 


\section{MÉTHODOLOGIE}

Nous avons opté pour une démarche comparative entre deux communes de standing contrasté, considérant que celle-ci peut contribuer à l'identification précise des facteurs de la différenciation spatiale d'accès au service d'enlèvement des ordures.

Comme commune de haut standing, notre choix s'est porté sur la commune de Cocody, deuxième commune la plus riche de la ville en termes de budget $42700 \mathrm{Fcfa} / \mathrm{hab}$. en 1998 (De Dianous, 1998) -, après le CBD que constitue la commune du Plateau. Ancien quartier blanc de l'ex-colonie, la commune de Cocody abrite la haute classe ivoirienne et la majorité des cadres expatriés. En vertu de son statut de commune périphérique, où les investissements dans les équipements font défaut et qui accueille les populations marginalisées, en grande partie immigrées, la commune d'Abobo sera étudiée au titre de commune de standing faible. Avec un budget de 2720 Fcfa/hab. en 1998, sa situation et ses origines en font, de fait, la commune la plus pauvre de la ville (De Dianous, 1998). La méthode mobilisée combine recherche bibliographique, entretiens avec les acteurs-clé du système, enquêtes et observations de terrain dans les deux communes.

L'objectif des enquêtes de terrain est de comprendre la différence de comportement face aux ordures ménagères chez les habitants des deux communes, et surtout, à l'instar de la démarche opérée par Meunier-Nikiema (2007), d'aboutir à une analyse de l'offre, pour laquelle les données statistiques sont inexistantes. Ces données sont ensuite liées aux observations relatives aux caractéris- tiques socio-économiques des quartiers et de leur population, et au fonctionnement du système d'enlèvement des ordures ménagères. Nous avons analysé trois quartiers dans chaque commune étudiée. Partant de l'affirmation que «les contrastes de l'habitat se superposent assez bien aux différents modes d'élimination des déchets» (Meunier-Nikiema, 2007), les quartiers analysés ont été choisis sur base de différents types d'habitat. Ainsi, dans la commune d'Abobo, les trois quartiers choisis sont respectivement un quartier d'habitat économique (Sogefiha), construit par une société immobilière d'Etat, un quartier ancien d'habitat en cours (Gagnoa Gare) - habitat traditionnel des villages ivoiriens composé d'une cour commune autour de laquelle sont construites plusieurs habitations - et un quartier périphérique d'habitat précaire urbanisé récemment de façon anarchique (Agouéto). Concernant la commune de Cocody, les quartiers choisis sur base des mêmes critères sont Riviera Palmeraie, quartier de nouvelles constructions de standing mité d'habitat précaire, le quartier des Caféiers, enclave résidentielle de jeunes cadres construite par opérations groupées, et enfin le village traditionnel Ebrié d'Anono, englobé par l'urbanisation (fig. 1).

Quinze enquêtes ont été administrées de manière directe dans chaque quartier, selon un schéma de dispersion aléatoire, au domicile, prioritairement aux femmes. Celles-ci sont en effet considérées plus centrales par rapport au processus d'élimination des ordures; ce sont elles, plus que leur mari, qui détermineront le comportement de leurs enfants ou de leurs domestiques. 


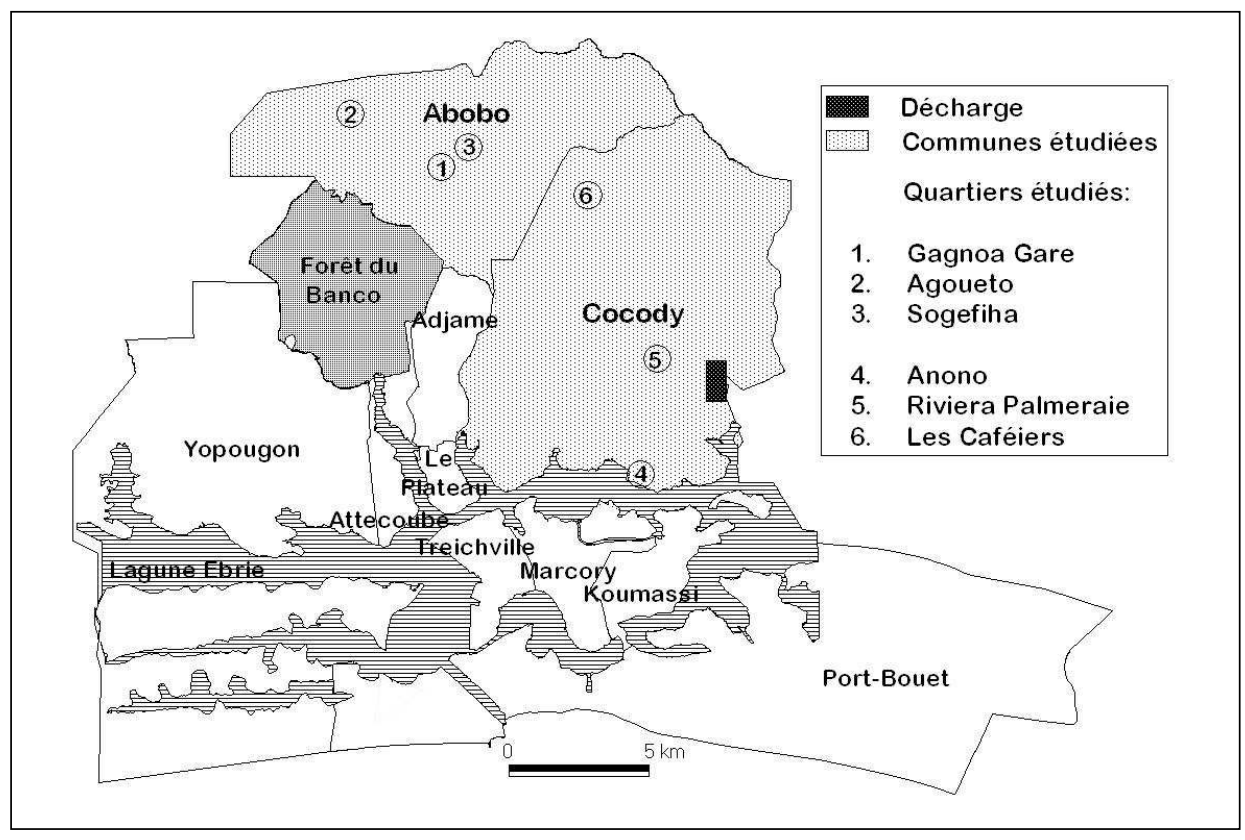

Figure 1. Espace étudié.

Source: Henry, 2009

\section{L'ENLĖVEMENT DES ORDURES À ABIDJAN}

Rappelons brièvement les différents modes d'évacuation des ordures actuellement utilisés à Abidjan (fig. 2).

Comme dans la plupart des villes africaines, la filière de gestion des ordures à Abidjan se fait en trois étapes: la précollecte, la collecte et le traitement. Dans les $P E D$, les deux premières étapes n'excèdent souvent pas un ramassage de 60\% des déchets produits (Ngnikam et Tanawa, 2007) - contre plus de $90 \%$ dans les pays développés. On cite le taux de 51\% de collecte effective des déchets produits actuellement à Abidjan (ANASUR, 2009). La décharge de la ville est située à l'extrême Est de la commune de Cocody, à proximité du petit village Ebrié d'Akouédo rattrapé par l'urbanisation (fig. 1).

La précollecte est l'ensemble des opérations d'évacuation des déchets, depuis leur lieu de production jusqu'au lieu de prise en charge par les véhicules de collecte (coffres à ordures, postes de groupa- ge). Il existe deux modes de précollecte: - l'apport volontaire des ordures par les habitants vers les espaces de coffres aménagés;

- la précollecte en porte-à-porte, où les précollecteurs récoltent les ordures aux portes des habitations et les acheminent à l'aide de charrettes en bois ou de voiturettes vers des espaces de coffres à ordures ou vers des postes de groupage (espaces de coffres plus volumineux et aménagés de ponts permettant aux précollecteurs de déverser leurs charrettes), d'où elles sont ensuite transférées à la décharge par les entreprises de collecte. A l'origine destinée aux seuls secteurs inaccessibles aux véhicules de collecte, la précollecte s'est ensuite étendue à tout le territoire d'Abidjan pour pallier les manquements des services de collecte en porte-à-porte.

La collecte est l'ensemble des opérations qui consistent à récupérer les déchets 
aux espaces de coffres et postes de groupage, et à les acheminer vers la décharge. Lorsque l'état de la voirie le permet, la collecte peut être effectuée en porte-à-porte par les camions de collecte. Ce mode de collecte est rare actuellement, mais était plus courant avant les années 90 .

Depuis la période coloniale, le système de gestion qui prévaut à Abidjan consiste en contrats de délégation de service, c'est-à-dire à la concession du service, par les autorités, à des entreprises privées qu'elles rémunèrent.

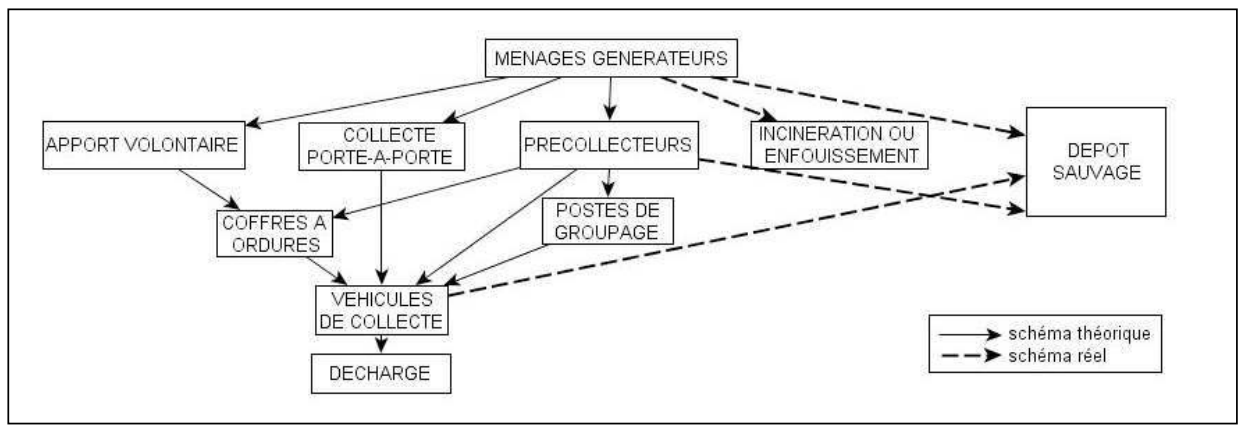

Figure 2. Organisation de l'enlèvement des ordures à Abidjan.

Source: Henry, 2009

\section{OBSERVATIONS}

Le tableau 1 présente les principaux résultats obtenus à travers les enquêtes et observations de terrain dans les différents quartiers étudiés.

A travers l'analyse des différents quartiers, on comprend la diversité des situations réelles possibles en matière de système d'enlèvement des ordures ménagères. Les inégalités socio-spatiales d'accès au service sont indubitables, tant au niveau de la collecte que de la précollecte: dans la commune de Cocody, les précollecteurs sont généralisés et il subsiste même un service partiel de collecte en porte-à-porte. Alors qu'à Abobo, non seulement la collecte en porte-à-porte est inexistante, mais la précollecte fait également défaut dans plusieurs quartiers et la collecte classique aboutit parfois à la création de dépôts sauvages par les véhicules de collecte eux-mêmes.

Cette situation ne laisse que peu d'alternatives à la population de certains quartiers d'Abobo: l'apport volontaire aux coffres trop rares (au nombre de 18 pour l'ensemble de la commune, soit moins d'un coffre pour $4 \mathrm{~km}^{2}$ ) et insuffisamment vidés, ou le dépôt sauvage, dont les localisations deviennent parfois ancrées dans les habitudes. Ils peuvent aussi servir de manifestation ostentatoire de mécontentement contre un service d'enlèvement des ordures qui ne les prend pas en compte, par exemple lorsqu'ils apparaissent en plein milieu des routes. Les enquêtés considèrent en général que la situation en matière de salubrité empire à toutes les échelles, et regrettent la situation d'autrefois, lorsque la collecte en porte-à-porte était plus fréquente et plus étendue.

Les données de collecte obtenues à I'ANASUR (Agence NAtionale de Salubrité URbaine), organe actuellement en charge de l'enlèvement des ordures d'Abidjan, complètent et confirment ces observations (tableau 2).

Ces constats établis, nous allons à présent tenter de comprendre comment et pourquoi une telle diversité d'accès au service d'enlèvement des ordures ménagères s'est mise en place au sein de la ville. 


\begin{tabular}{|c|c|c|c|c|c|c|}
\hline 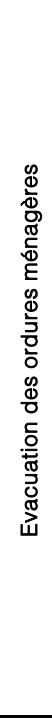 & 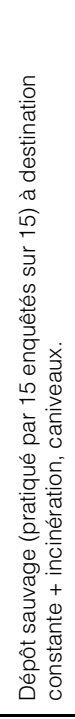 & 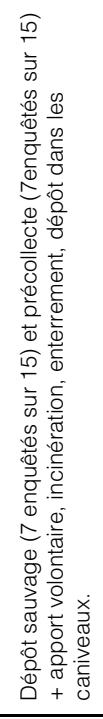 & 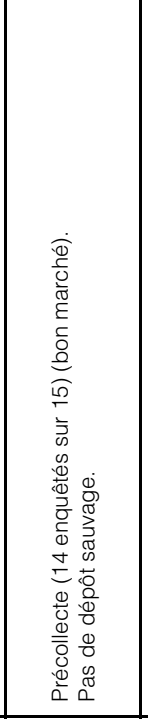 & 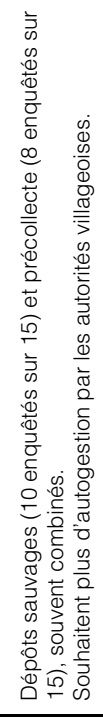 & 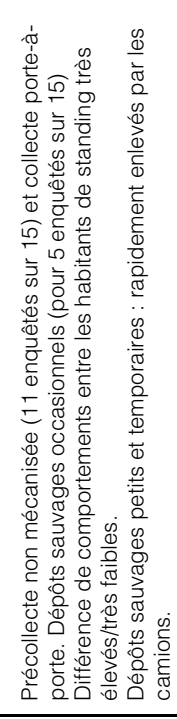 & 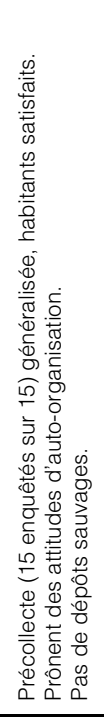 \\
\hline 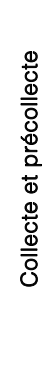 & 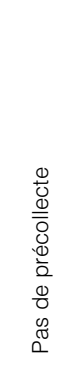 & 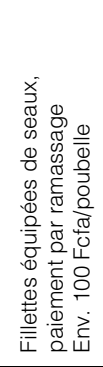 & 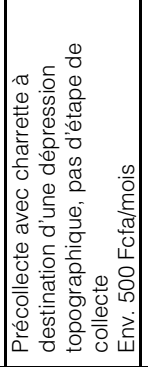 & 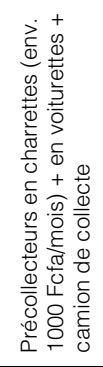 & 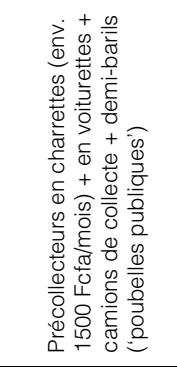 & 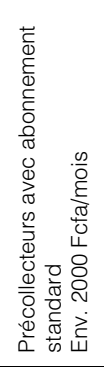 \\
\hline 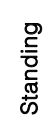 & 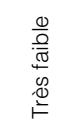 & 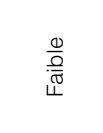 & $\begin{array}{l}\text { D. } \\
\text { Dे }\end{array}$ & $\begin{array}{l}\frac{0}{00} \\
\frac{0}{\tilde{w}}\end{array}$ & 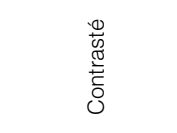 & $\frac{.}{\tilde{W}}$ \\
\hline 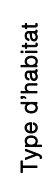 & 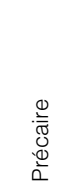 & 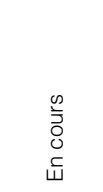 & 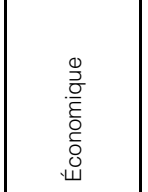 & 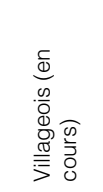 & 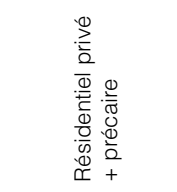 & 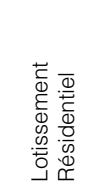 \\
\hline 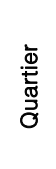 & $\begin{array}{l}\frac{9}{0} \\
\stackrel{0}{0} \\
\stackrel{9}{8}\end{array}$ & 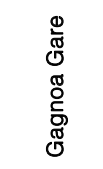 & 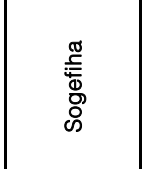 & $\begin{array}{l}\frac{8}{0} \\
\frac{0}{4}\end{array}$ & 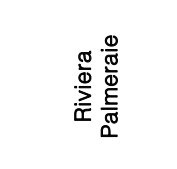 & 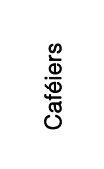 \\
\hline 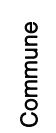 & & $\begin{array}{l}\stackrel{0}{0} \\
\frac{0}{4}\end{array}$ & & & $\begin{array}{l}\text { 하 } \\
\text { ठ․ }\end{array}$ & \\
\hline
\end{tabular}

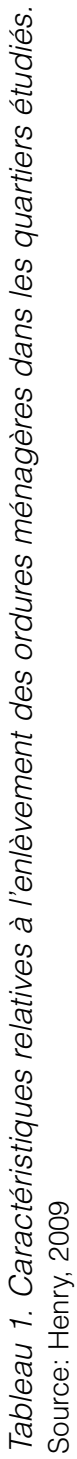

BELGEO • 2009 • 3-4 


\begin{tabular}{|c|r|r|r|}
\hline Communes & $\begin{array}{c}\text { ordures à } \\
\text { collecter }\end{array}$ & $\begin{array}{c}\text { ordures } \\
\text { collectées }\end{array}$ & $\begin{array}{c}\text { taux de } \\
\text { collecte }\end{array}$ \\
\hline Abobo & 104300 t. & 23900 t. & $23 \%$ \\
\hline Cocody & 41100 t. & 29300 t. & $71 \%$ \\
\hline
\end{tabular}

Tableau 2. Collecte des déchets de mai à septembre 2008.

Source: ANASUR, 2009

\section{LA GESTION DU SERVICE: UN ÉLÉMENT CRUCIAL}

Parmi la diversité des facteurs qui concourent à produire une telle situation, la gestion du service par les autorités publiques prend une place prépondérante. Celle-ci a évolué au rythme des avancées de la Côte d'Ivoire dans le processus de décentralisation dans laquelle elle est engagée depuis le début des années '80. Depuis lors, la compétence est déléguée progressivement à un niveau de gestion toujours plus local. Ce n'est que récemment que celle-ci est recentralisée au niveau de l'ANASUR.

On peut scinder la chronologie de la gestion des ordures à Abidjan en quatre grandes périodes:

- 1953 - 1980: la gestion par l'Etat de Côte d'Ivoire:

- 1980 - 2002: la gestion par la Ville d'Abidjan;

- 2002 - 2007: la gestion par le District d'Abidjan et les communes;

- Depuis 2007: la gestion recentralisée au niveau du Ministère de la Ville et de la Salubrité.

\section{LA GESTION PAR L'ETAT DE CÔTE D'IVOIRE}

A partir de 1953, l'enlèvement des ordures de la commune d'Abidjan et de quelques autres capitales africaines est concédé à une société privée, la SITAF, filiale d'une société française spécialisée dans la production de matériel de transport et de traitement. Elle est rémunérée en fonction de simples déclarations du tonnage transporté et de la distance parcourue. En 1971, une taxe sur la consom- mation domestique d'électricité est introduite pour financer le service. Les tarifs, fixés à 2,5 Fcfa/kWh n'ont jamais suffi au financement total de l'enlèvement des ordures ménagères et les recettes n'ont jamais été reversées intégralement par la compagnie d'électricité.

\section{LA GESTION PAR LA VILLE D'ABIDJAN}

En 1980, la commune d'Abidjan prend le statut de Ville, composée de dix communes. La Ville d'Abidjan se voit accorder la responsabilité de l'enlèvement des ordures ménagères, dont les sources de financement proviennent en majorité des contributions des communes. Durant toute cette période et jusqu'en 2002, le modèle financier qui prévaut est celui de la péréquation: chaque commune verse un pourcentage de ses recettes à la Ville pour l'enlèvement des ordures. La contribution que verse une commune varie entre 10 et $55 \%$ de ses recettes, en fonction des recettes moyennes par habitant dont elle dispose, assurant ainsi une redistribution des richesses.

Malgré la croissance explosive de la population durant ces décennies, le service d'enlèvement des déchets fonctionne relativement bien et semble plus égalitaire. Le mode de collecte majeur est le porte-à-porte. Le taux de collecte est de $75 \%$ en 1989; il ne cessera pas de chuter par la suite (Ministère de l'environnement et du cadre de vie, 2001).

A la fin des années 80, la crise économique s'aggrave et l'Etat ne rémunère pas correctement les entreprises nationales. 
La SITAF, accusée de surfacturation, se retire et les autorités de la Ville reprennent temporairement en charge l'enlèvement des ordures. Mais le matériel dont elles disposent est vétuste et insuffisant; le secteur de l'enlèvement des ordures est en crise et les dépôts illégaux se multiplient. C'est au cours de cette période qu'un accès inégal au service commence à se manifester au sein de la ville. La collecte est effectuée prioritairement dans les communes centrales aisées, alors que, dans les autres, la population n'a d'autre choix que de s'organiser pour accomplir elle-même l'enlèvement de ses ordures (Attahi, 2002). C'est ainsi que naît le secteur de la précollecte, dont le coût incombe aux ménages sur base de contrats informels, et qui s'étend peu à peu au-delà des seuls quartiers inaccessibles aux véhicules de collecte.

En 1992, la nouvelle entreprise prestataire, ASH International, remporte de manière houleuse le contrat de l'enlèvement des ordures ménagères d'Abidjan. Le matériel vétuste continue à causer de nombreuses pannes qui précipitent l'avènement d'une des plus grandes crises des ordures: le taux de collecte passe à $55 \%$ en 1994, malgré le décaissement, cette année-là, de $61 \%$ du budget de la Ville (Sané, 2002). A cette époque, plus de $30 \%$ des Abidjanais ont un accès mauvais ou moyen au service d'enlèvement des ordures (indiqué par l'absence de collecte en porte-à-porte dans le secteur et l'absence de coffres à ordures, vidés régulièrement, à moins de $500 \mathrm{~m}$ du domicile). Ce pourcentage monte à $64,1 \%$ à Abobo, qui obtient la pire performance (DCGTx, Amélioration des services urbains dans les quartiers souséquipés, juin 1995). La répartition des coffres à ordures de la ville est également discutable (tableau 3).

Les bilans nationaux commencent à souligner l'importance de la précollecte (étape indispensable dans $20 \%$ des quartiers), qui demeure informelle et mal reconnue, et la volonté de l'intégrer de manière plus officielle dans la filière de gestion des ordures.

Les délais de collecte sont rallongés malgré une charge croissante pour les communes. Celles qui peuvent se le permettre complètent alors ponctuellement l'action d'ASH en louant ou en achetant des bennes. II n'est pas étonnant dès lors que plusieurs communes réclament l'auto-gestion des ordures de leur territoire.

\section{LA GESTION PAR LE DISTRICT ET LES COMMUNES}

En 2001, la Ville d'Abidjan est érigée en District, défini comme une collectivité territoriale dotée de la personnalité morale et de l'autonomie financière, placée sous la tutelle de l'Etat. II est composé des 10 communes de l'ex-Ville et de 3 sous-préfectures: Bingerville, Anyama et Songon. Les gouvernements locaux assument de plus en plus le contrôle des affaires locales. Durant cette période, le District d'Abidjan est chargé de la collecte des ordures et de la gestion de la décharge, et les communes sont chargées d'assurer la précollecte.

Malheureusement, travers courant accompagnant les processus de décentralisation, les budgets des collectivités locales ne croissent pas pour autant. La mesure se résume alors au transfert de la crise financière vers ces collectivités locales («décentralisation de la pénurie»). La suspension des investissements et les difficultés de trésorerie rencontrées dans un climat socio-politique très instable bouleversent la gestion des déchets.

De plus, les rémunérations des entreprises prestataires de collecte sont indépendantes cette fois de la distance à la décharge. Cela favorise l'abandon de

\begin{tabular}{|l|c|c|c|c|}
\hline Communes & Coffres $14 \mathrm{~m}^{3}$ & Coffres $7 \mathrm{~m}^{3}$ & Coffres $3 \mathrm{~m}^{3}$ & Coffre par habitant $\left(\mathrm{m}^{3}\right)$ \\
\hline Cocody & 7 & 6 & 35 & 0,014 \\
\hline Abobo & 7 & 4 & 27 & 0,005 \\
\hline
\end{tabular}

Tableau 3. Coffres à ordures par commune en 1993.

Source: Attahi, 2002 


\begin{tabular}{|l|c|c|}
\hline Communes & Collecte 2005 $(\mathrm{t})$ & Collecte 2005 (t/hab.) \\
\hline Abobo & 118700 & 0,15 \\
\hline Cocody & 123500 & 0,39 \\
\hline
\end{tabular}

Tableau 4. Collecte des déchets dans les deux communes en 2005.

Source: Mairie d'Abidjan et commune de Cocody, 2006

certaines communes périphériques (comme Abobo) au profit de communes proches de la décharge, comme Cocody. Les défaillances financières pèsent à tous les niveaux et les infrastructures construites par le District demeurent insuffisantes. Les mairies qui en ont les moyens se substituent alors tant bien que mal au District, en disposant des coffres à ordures là où des dépôts sauvages sont constatés.

En transférant la précollecte aux communes, les solidarités intercommunales qui réduisaient les disparités de budget pour la gestion des ordures sont brisées. C'est alors que les disparités des budgets qui affectent les communes prennent une importance nouvelle. Entre 70 et $90 \%$ des recettes communales proviennent de l'impôt foncier et des patentes. Des communes comme Abobo ou Yopougon, dont le budget par habitant et par an est (en 1998) de 2720 et 4370 Fcfa, ne peuvent rivaliser avec celles de Cocody ou du Plateau qui disposent respectivement de 42700 et près de 300000 Fcfa par habitant et par an, soit un rapport de 1 à 100 entre les budgets extrêmes (De Dianous, 1998). Mais cette disparité budgétaire n'est pas la seule cause d'une gestion si hétérogène; elle devient parfois aussi une raison justifiant trop facilement les inactions et les faiblesses politiques. Le rapport des compétences des gestionnaires est malheureusement parfois du même ordre que celui des budgets.

Ainsi, les gestionnaires cocodyens ont montré une profonde attention à la salubrité de la commune, qui constitue une des priorités principales de leurs fonctions. Ils ont imposé à la société de précollecte retenue d'oeuvrer avec les associations de jeunes précollecteurs en place et ont effectué un travail important de structuration et d'encadrement de ces jeunes travailleurs, par exemple en les rendant responsables d'une zone précise, ou en organisant avec eux des réunions fréquentes afin d'assurer un suivi strict de leurs activités, besoins et problèmes rencontrés. Toutes ces initiatives ont amené une forte responsabilisation et sensibilisation des précollecteurs. Aujourd'hui, après le transfert de la compétence à l'ANASUR, ces réalisations restent acquises et les consignes restent en vigueur. C'est un facteur crucial supplémentaire permettant de comprendre la meilleure salubrité de la commune de Cocody.

En août 2006, le tristement célèbre scandale des déchets toxiques à Abidjan entraîne la fermeture de la décharge pendant deux mois durant lesquels la situation sanitaire de la ville est accablante. Le gouverneur de District suspend inopinément les contrats de plusieurs sociétés prestataires et redéfinit un nouveau zonage, perturbant à nouveau la stratégie mise en place.

\section{LA GESTION RECENTRALISÉE AU NIVEAU DU MINISTĖRE DE LA VILLE ET DE LA SALUBRITÉ}

Depuis décembre 2007, au vu du constat d'échec qu'accusent les municipalités dans la gestion des ordures ménagères, le gouvernement confie la gestion de la filière des déchets du pays à l'ANASUR, dont les ressources sont alimentées par des dotations du budget de l'Etat, des taxes et parts des contributions foncières. C'est donc une étape de recentralisation qui paraît aller dans le sens opposé à la décentralisation instaurée dans la Constitution.

Un des changements capitaux introduits par le transfert de la responsabilité à l'ANASUR est le rapport au secteur de la précollecte. Alors que, sous la gestion 
municipale, les précollecteurs étaient subventionnés en fonction du tonnage par l'intermédiaire des entreprises prestataires (en plus de la cotisation des ménages), à présent, le système de gestion des ordures ne tient plus compte des précollecteurs. L'ANASUR n'a pas été mandatée pour la gestion de la précollecte et ne prévoit donc pas de budget pour ce secteur. Les entreprises prestataires font alors de même, ou du moins disposent de toute la latitude nécessaire pour gérer la relation qu'elles désirent avec les coopératives de précollecteurs. Pire, alors qu'auparavant elles subventionnaient les précollecteurs au tonnage, considérant l'activité comme une sorte de sous-traitance, les entreprises considèrent à présent que les précollecteurs les dessaisissent d'une partie de leur marché; elles leur font alors payer une contribution à chaque charrette déposée au coffre à ordures. Ces coûts supplémentaires, ajoutés aux files d'attente aux coffres, ne peuvent qu'encourager les précollecteurs à se débarrasser clandestinement des ordures. C'est un point capital pour expliquer la constitution de dépôts illégaux dans la ville.

Mais la conséquence la plus grave de cette absence de prise en compte de la précollecte est sans nul doute l'augmentation de la charge financière répercutée sur les ménages. Ce qui fait surgir tous les effets qu'engendre une privatisation: introduction de logiques marchandes, inégalités d'accès, privilèges des quartiers les plus solvables et abandon des autres. Cela confirme le processus de fragmentation urbaine, avec création d'espaces solvables à desserte de qualité et contournement des secteurs incapables de se l'offrir. On comprend alors l'abandon par les précollecteurs de certains quartiers d'Abobo comme Agouéto et Gagnoa Gare.

De plus, alors qu'on aurait pu croire que la recentralisation de la compétence gommerait les différences de qualité du service entre les deux communes, on constate à l'inverse que ce changement ne fait qu'aggraver ces disparités, en raison d'une gestion très hétérogène par I'ANASUR. En effet, la commune de
Cocody, en plus d'être considérée comme une vitrine de la ville, abrite la majorité des politiciens du pays et une grande partie des hauts cadres d'Abidjan. Cette population est plus importante aux yeux des gestionnaires de I'ANASUR, qui accordent plus d'attention et d'énergie en cas de plainte ou de problème de salubrité provenant de la commune de Cocody. Cette gestion partiale s'illustre le plus clairement dans le recrutement des entreprises prestataires. L'ANASUR a rapidement lancé un appel d'offre pour la collecte des communes regroupées en zones. La commune de Cocody a été affectée à la société Cleanbor-Cl, et celle d'Abobo, à la société Intercor. La qualité des sociétés diffère profondément. Cleanbor-Cl a remporté trois grands prix internationaux en 2008 et jouit d'une réputation internationale irréprochable. Elle dispose d'un meilleur matériel automobile et d'un capital important qui lui permet de continuer à fonctionner même en cas de retard de paiement par l'ANASUR. L'enlèvement des ordures de la commune d'Abobo a été attribué à Intercor, une petite entreprise inconnue jusque là, et qui doit pourtant collecter près du quart des ordures du District. Son capital est réduit et elle ne détient pas non plus réellement les moyens techniques, financiers et humains qu'elle a déclaré posséder lors de sa réponse à l'appel d'offre. Son parc automobile est insuffisant et vieillissant, et les gérants manquent de capacités de gestion.

Dans le service d'enlèvement des ordures ménagères, la gestion est dès lors un facteur primordial pour réguler l'égalité socio-spatiale intra-urbaine. Qu'il s'agisse de précollecte ou de collecte, que la gestion soit communale ou plus centralisée, les décisions prises par les gestionnaires peuvent fortement accentuer les inégalités d'accès au service. Néanmoins, d'autres facteurs interviennent pour complexifier ce schéma, comme la distance moyenne à la décharge, la topographie, les voiries, et surtout des facteurs d'ordre socio-économique comme la solvabilité de la population (par le biais de la précollecte), et le comportement des habitants. 
La ville d'Abidjan présente des caractéristiques d'urbanisation communes à la majorité des grandes villes des pays en développement. L'extension spatiale rapide de l'agglomération, gonflée par une croissance démographique explosive, n'a pas été suivie au même rythme par le développement des services publics. La ségrégation socio-spatiale inscrite depuis bien longtemps dans le territoire abidjanais structure un accès différentiel aux services urbains.

De la même façon, le service d'enlèvement des ordures ménagères est sujet à de nombreuses critiques depuis plusieurs décennies. II ne s'est pas adapté au rythme de croissance de la population abidjanaise. II en résulte des carences importantes, qui se concrétisent par une mise en décharge de seulement la moitié des déchets produits, et par la multiplication des dépôts sauvages dans la ville. Nous avons constaté que l'accès à ce service d'enlèvement des ordures ménagères comporte des contrastes spatiaux profonds, qui se superposent bien aux contrastes socio-économiques des différents quartiers et semblent s'accentuer au cours du temps, au gré de l'évolution de la gestion du service. Nous sommes bien en présence d'un processus de fragmentation urbaine en petites unités territoriales, avec création de zones de desserte de première classe et isolement d'autres.

L'évolution du service se caractérise par les transferts successifs de la responsabilité de sa gestion à différents niveaux de pouvoir, en parallèle avec un mouvement dialectique de centralisation, décentralisation, recentralisation. La chronologie de la gestion du service montre une diversité de systèmes de fonctionnement, qui semblent en avoir progressivement accentué les inégalités d'accès.

Un tel processus de fragmentation urbaine est souvent relié aux modalités courantes de mise en oeuvre des réformes dans les services en réseau (privatisations et recherche de profit, qui conduisent les entreprises à privilégier la clientè- le aisée et à contourner la clientèle peu solvable) (Graham, 2000). Ici, en revanche, ce processus prend place malgré un système de concession du service par les autorités à des entreprises privées. On ne peut donc pas (du moins, en ce qui concerne la collecte) l'attribuer à un processus de privatisation dans lequel les inégalités d'accès seraient dues à des inégalités de pouvoir d'achat des ménages. Et ce processus se poursuit malgré une gestion à présent recentralisée. On ne peut donc pas non plus l'imputer à des budgets communaux différentiels, qui engendreraient une qualité différenciée du service selon les communes. Enfin, on ne peut l'imputer à une gestion clairement inégalitaire, qui limiterait les actions de la municipalité en matière de collecte à une partie de la ville, comme c'est le cas dans certaines capitales africaines. Ici, le système semble, en théorie, potentiellement égalitaire. II masque toutefois d'autres mécanismes inégalitaires plus vicieux, comme la contractualisation des autorités avec des sociétés plus performantes dans les communes riches. Cette différence acquiert une importance plus vive dans un contexte de retard de paiement des entreprises par l'Etat, et témoigne d'une attention globale inégale envers les différentes communes et leur population. De plus, conformément aux tendances de désengagement de l'Etat par rapport aux services publics, la précollecte est désormais exclue de la considération des autorités - dans les mandats, et donc dans les budgets - laissant les logiques marchandes envahir le secteur. Les précollecteurs ne sont plus rémunérés que par les ménages, ce qui provoque, dans ce secteur informel, les mêmes conséquences qu'une privatisation : la solvabilité des ménages devient une condition d'existence de la précollecte, qui délaisse alors les quartiers les plus pauvres.

Ces inégalités dans l'accès à l'enlèvement des ordures ménagères se manifestent à tous niveaux, de la précollecte à la collecte. Une pluralité de facteurs est à la 
source de cet état de fait; ils ne sont pas uniquement d'ordre financier ou gestionnaire, mais aussi d'ordres historiques, socio-économiques et physiques. Face à tous ces facteurs, on ne peut imaginer résoudre la situation rapidement, car chacun de ceux-ci est empreint d'une certaine inertie. Il est également nécessaire de considérer le service non pas en vase clos, mais lié à d'autres éléments, comme le renforcement des voiries sans lequel une amélioration de la collecte en porteà-porte dans les communes périphériques ne serait pas envisageable.

Enfin, afin d'améliorer la gestion des ordures ménagères dans les pays en développement, on prône parfois une décentralisation plus aboutie de la compétence ainsi que des budgets. Toutefois, une (re-)décentralisation du service de gestion des ordures au niveau communal à Abidjan exigerait la réintroduction d'une forme de solidarité inter-communale, comme la péréquation, sans laquelle l'achèvement du processus de décentralisation (sans plus de reversements financiers de la part de l'Etat) ne serait bénéfique que pour les communes riches comme Cocody, qui disposent d'un véritable potentiel économique. Pour les autres, comme Abobo, une décentralisation plus aboutie dans ce sens aurait des effets négatifs, comme ce fut le cas dans le passé, car la faiblesse des ressources ne permettrait pas aux municipalités d'honorer leurs obligations, ce qui n'aurait pour effet que de les décrédibiliser davantage aux yeux de leurs habitants.

\section{BIBLIOGRAPHIE}

- ACHANKENG E. (2003), "Globalization, urbanization and Municipal solid waste management in Africa", actes de la conférence Africa on a global stage, African studies association of Australasia and the Pacific, $21 \mathrm{p}$

- ANASUR (2009), Données de collecte des ordures dans les communes d'Abidjan, Abidjan, 13 p.

- ATTAHI K. (2002) «Abidjan, Côte d'Ivoire", in ONIBOKUN A. G. et al. (2002), La gestion des déchets urbains. Des solutions pour l'Afrique, CRDIKarthala, Paris, pp. 17-51

- COURET D. et METZGER P. (2002), «La ville durable côté Sud: entre utopies et pratiques», in MARTIN J.-Y. (2002), Développement durable? Doctrines, pratiques, évaluations, IRD, Paris, pp. 161182

- DCGTX (1995), Amélioration des services urbains dans les quartiers souséquipés, Abidjan, $26 \mathrm{p}$.

- DE DIANOUS S. (1998), «Abidjan», Marchés tropicaux et méditerranéens, hors série, $78 \mathrm{p}$

- DORIER-APRILL E., JAGLIN S. et al. (2002), Gérer la ville: entre le global et le local, éditions de l'Aube, Paris, 198 p.

- GRAHAM S. (2000), "Constructing premium network spaces: reflections on infrastructure networks and contemporary urban development", International Journal of Urban and Regional Research, vol. 24, n¹, pp. 183-200

- HENRY A. (2009), Les inégalités sociospatiales d'accès aux services urbains dans les pays en développement: le cas de l'enlèvement des ordures ménagères à Abidjan, Mémoire de Master en Géographie, Université Libre de Bruxelles, $93 \mathrm{p}$.

- MARVIN S. et GRAHAM S. (2001), Splintering Urbanism: Networked infrastructures, technological mobilities, and the urban condition, Routledge, Londres, $479 \mathrm{p}$

- MBOUMBA A. (2007), «Gestion urbaine et équité socio-spatiale: les inégalités dans les services de base à Libreville (Gabon)», L'Espace Géographique, vol. 36. n², pp. 131-140

- MEUNIER-NIKIEMA A. (2007), «Géographie d'une ville à travers la gestion de ses déchets. Ouagadougou (Burkina-Faso)», Mappemonde, vol. 87, $\mathrm{n}^{\circ} 3,15 \mathrm{p}$.

- MINISTÈRE DE L'ENVIRONNEMENT ET DU CADRE DE VIE (2001), Gestion durable des déchets. Stratégie et programme national, Direction de l'environnement et du cadre de vie, Abidjan, 56 p. 
- NGNIKAM E. et TANAWA E. (2007), Les villes d'Afrique face à leurs déchets, UTBM, Belfort-Montbéliard, $280 \mathrm{p}$.

- SANE Y. (2002), «La gestion des déchets à Abidjan: un problème récurrent et apparemment sans solution", AGEAM/Ragée, vol. 4, n¹, pp. 13-22

Amandine Henry

henry.amandine@gmail.com

manuscrit déposé en novembre 2009; révisé en mai 2010 